\title{
Incomplete preferences and rational intransitivity of choice ${ }^{\text {th }}$
}

\author{
Michael Mandler \\ Department of Economics, Royal Holloway College, University of London, Egham Surrey TW20 OEX, UK
}

Received 2 August 2002

Available online 18 August 2004

\begin{abstract}
Do violations of classical rationality theory imply that agents are acting against their self-interest? To answer this question, we investigate whether completeness and transitivity necessarily hold when agents choose outcome rationally - that is, their choice sequences do not lead to dominated outcomes. We show that, because of the danger of money pumps and other manipulations, outcome rationality implies that agents must have transitive psychological preferences. Revealed preferences, on the other hand, must be complete since agents can be forced to choose from any set of options. But these justifications of transitivity and completeness cannot be combined. We show that if psychological preferences are incomplete then revealed preferences can be intransitive without exposing agents to manipulations or violating outcome rationality. We also show that a specific case of nonstandard behavior, status quo maintenance, is outcome-rational in the simple environments considered in the experimental literature, but not in more complex settings.
\end{abstract}

(c) 2004 Elsevier Inc. All rights reserved.

JEL classification: D11; D81

Keywords: Incomplete preferences; Revealed preference; Transitivity; Status quo maintenance; Behavioral economics

\footnotetext{
This paper is a much revised version of the formal parts of [Mandler, M., 1998. The economics of incomplete preferences. Mimeo. Harvard University, Cambridge], whose informal sections appear in [Mandler, M., 2004. Status quo maintenance reconsidered: changing or incomplete preferences? Econ. J. 114, 518-535.].

E-mail address: m.mandler@rhul.ac.uk.
}

0899-8256/\$ - see front matter ㅇ 2004 Elsevier Inc. All rights reserved. doi:10.1016/j.geb.2004.02.007 


\section{Introduction}

Despite copious evidence discrediting the theory of rational choice (see Camerer, 1995 and Rabin, 1998), the theory continues to wield considerable authority. One reason is that the core rationality axioms, completeness and transitivity, are thought to follow from selfinterest; agents who violate those axioms supposedly expose themselves to manipulation or experience diminished welfare. This paper analyzes the link between completeness and transitivity and the assumption of rational self-interest: do the completeness and transitivity axioms follow from the more fundamental assumption that agents' choices not lead to less preferred outcomes? Although some casual arguments address this question, economic theory usually just labels complete and transitive preferences as rational. We instead take as our starting point that agents are outcome-rational, that is, their choice sequences do not lead to dominated outcomes. We will see that outcome-rational agents can always violate either completeness or transitivity: self-interest does not weed out violations of the traditional rationality model.

To analyze rationales for the completeness and transitivity axioms, we distinguish between agents' psychological preferences (their judgments about their welfare or wellbeing) and their choice behavior or "revealed preferences." Informal arguments for completeness and transitivity often switch back and forth between these two definitions of preference. Each axiom can be justified using one of the definitions of preference, but the two axioms taken jointly cannot be rationalized under either definition.

With the psychological definition of preference, the rationality of transitivity is easy to establish. A formalization of the famous money pump shows that agents with intransitive psychological preferences are not outcome-rational-they can be led into sequences of trades that diminish their initial endowment (Section 3). The completeness of psychological preferences, on the other hand, has no obvious justification. The primary argument for completeness instead relies on the choice definition of preference: since agents can always be compelled to choose from any pair of alternatives, revealed preferences must be complete. Can these cases for completeness and transitivity be combined? They could if psychological preference and choice necessarily coincided. But although one may always interpret an agent's choices as a set of psychological welfare judgments, if choice is not in reality guided by such welfare judgments-because those judgments are incomplete- then choice need not obey the consistency properties, such as transitivity, that rational psychological preferences must satisfy. Sen $(1973,1982,1997)$ and Levi (1986) offer further arguments for distinguishing between choice and preference.

If there is a case for why rational revealed preferences must be transitive, it rests on the claim that any intransitivity would expose agents to the same manipulations that accompany intransitive psychological preferences. Perhaps then outcome-rational agents must choose transitively and thus act as if they had complete and transitive psychological preferences. The drawbacks of intransitive psychological preferences do in fact have exact parallels in the theory of choice functions: if the revealed preference relation derived from an agent's choice function is intransitive, the agent will be open to a money-pump manipulation that reduces the agent's consumption (Section 4). But this apparent defect of intransitive revealed preference is an artifact of the traditional model of choice functions, 
which as a theory of static decision-making specifies only one set of choices for each set of alternatives.

We therefore introduce extended choice functions to model sequential choice and allow current choices to depend on prior choice sets and prior decisions. Our main result, Theorem 4, shows that intransitivity of choice is then consistent with outcome rationality: if an agent's psychological preferences are transitive and incomplete there exist extended choice functions that never lead to dominated outcomes but that generate intransitive revealed preferences. Hence, although one can use revealed preferences to assemble a complete ordering from an incomplete psychological preference relation, the ordering can be intransitive-but not irrational in the sense of leading to dominated outcomes. Completeness and transitivity cannot therefore be justified simultaneously: outcome-rational psychological preferences must be transitive but can be incomplete, while outcome-rational revealed preferences must be complete but need not be transitive.

We must distinguish between an agent's psychological preferences and his or her choices because we need an independent gauge of welfare to test an agent's outcomerationality. But each psychological preference concept we use, including indifference and incompleteness, has distinct behavioral implications and thus complies with ordinalist methods. In behavioral terms, an agent weakly prefers $x$ to $y$ when the agent is always willing to accept $x$ in exchange for $y$, and accordingly $x$ is indifferent to $y$ when the agent is always willing both to switch from $x$ to $y$ and from $y$ to $x$. In contrast, incomplete preference between $x$ and $y$ holds if the agent is only sometimes willing to switch from $x$ to $y$ or from $y$ to $x$ (see Section 7 for precise statements).

A willingness sometimes to choose $x$ over $y$ and sometimes to choose the reverse can help an agent who cannot rank $x$ and $y$ to avoid manipulation and achieve outcome rationality. This flexibility can also give a normative rationale for status quo maintenance (SQM), a common pattern of nontraditional decision-making that underlies the endowment effect, loss aversion, and the willingness to pay-willingness to accept disparity. If an agent has transitive but possibly incomplete preferences, then SQM is outcome-rational in the simple choice environments usually considered in behavioral economics (Section 6). Since SQM can generate intransitive choices, it provides a simple and prominent example of the consistency of intransitivity and outcome rationality. SQM also illustrates the rationality advantage of refusing to switch between unranked bundles. On the other hand, status quo maintainers can experience diminished welfare in more complex environments. ${ }^{1}$ Our use of SQM is similar to the Bewley's $(1986,1987)$ models of choice with incomplete preferences, although our focus is on whether and when SQM reduces agents' welfare. See Mandler (2004) on the advantages of an incomplete preferences explanation of SQM compared to competing behavioral theories.

Incomplete preferences have an intermittent research history that has revived recently. Prior to Bewley, Aumann (1962), in the context of choice under uncertainty, was seminal and discussed when an incomplete preference relation could be represented by a utility function (on which see Richter, 1966 and Peleg, 1970). More recently, the representation

\footnotetext{
1 This result is consistent with our main theorem since even though SQM can expose agents to harm in complex environments, other patterns of choice, which also violate transitivity, are outcome-rational.
} 
question has reemerged using a tighter and more satisfactory definition of representation via sets of utility functions (Ok, 2002 and Dubra et al., 2001).

Cubitt and Sugden (2001) consider the consistency properties that choice functions satisfy when agents are assumed to be invulnerable to money pumps. Some of our results are similar, e.g., the role of the time-independence and continuity of choice functions for some consistency properties (cp. Theorem 3 below and Propositions 5 and 6 in Cubitt and Sugden, 2001). But we focus on the compatibility of arguments for completeness and transitivity, leading us to analyze preference relations rather than choice functions, and we consider a broad class of potential hazards of choice, not just money pumps.

\section{An example}

We skip precise definitions in this section and sketch the main argument in an example.

After we review the money pump argument for why rational psychological preferences must be transitive in Section 3, we take an agent's choice behavior as primitive and consider the revealed preferences that stem from these choices. Option $x$ is said to be weakly revealed preferred to $y$, or $x R y$, if and only if the agent, when facing some set of options that contains both $x$ and $y$, selects $x$. Let $P$ denote the strict revealed preference relation derived from $R$. Since, for any pair of options $x$ and $y$, we may confront the agent with the choice set $\{x, y\}$, the agent must either show $x R y$ or $y R x$, or both: revealed preferences are necessarily complete.

Should a rational agent have revealed preferences that are transitive as well? Consider an agent with incomplete psychological preferences over two goods: the agent strictly prefers bundles containing more of both goods but is unable to rank bundles that trade off good 1 against good 2. So, for instance, if bundle $x$ contains more of good 1 and less of good 2 than $y$ (see Fig. 1), then the agent does not rank $x$ and $y$. In each period, the agent chooses either from a set of options or from a set of alternatives to the agent's current selection. To avoid the fact that goods consumed at different dates are different commodities, suppose the agent consumes only when the series of offers terminates.

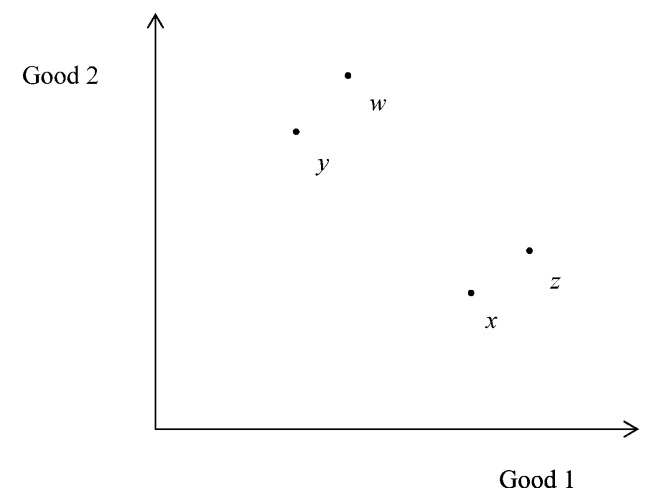

Fig. 1. Potential manipulations with incomplete preferences. 
To see the supposed case for why $R$ should be transitive, suppose the agent when facing a set of options $S$ that contains $x$ and $y$ (and no bundles dominating $x$ or $y$ ) selects $x$. Since $x$ and $y$ are not psychologically ranked, this behavior is rational when taken in isolation. Since the agent chooses $x$ from $S$, we have $x R y$. Let $z$ be a third bundle that contains more of both goods than $x$ but still less of good 2 than $y$. Since the agent is unable to rank $y$ and $z$, he or she might choose $y$ from some set $T$ that contains $y$ and $z$ (and again no dominating bundles). So $y R z$. Since $z$ is strictly psychologically preferred to $x$, a rational agent must exhibit $z P x$-if not, there would be some choice set at which agent selects $x$ when $z$ is available, which could leave the agent with a strictly inferior outcome. We therefore have the intransitivity $x R y, y R z, z P x$. Accordingly, we can offer the agent a money-pump-like sequence of decisions that lead from a superior to an inferior bundle: if the agent begins with $z$, he or she will select $y$ in exchange when offered the set of alternatives $T$, and then accept $x$ in exchange for $y$ when offered $S$. We will see in Section 4 that given a mild form of continuity any intransitivity of $R$ opens an agent to some such manipulation.

But this vulnerability to manipulation is misleading; it relies on the fact that we have supposed, following the choice function literature, that agents specify a single set of selections from any given choice set. In sequential environments in which agents make multiple rounds of decisions (and these are the only settings in which manipulation can arise), agents may want their current selections to depend on previous choice sets and decisions. Indeed, status quo maintainers employ just such a rule; they refuse all trades until offered an option that dominates the bundle they currently hold.

Status quo maintainers are immune to the above $z$-to- $x$ manipulation since they would decline at the first stage to switch from $z$ to $y$. Indeed, in certain simple environments, status quo maintenance (SQM) is outcome-rational and hence never open to the $z$-to- $x$ manipulation or any other manipulation (see Section 6). Since SQM readily exhibits intransitivity of choice, it thus furnishes an example of outcome-rational intransitivity. SQM also illustrates that sometimes refusing to exchange unranked bundles can be instrumental to achieving outcome rationality.

SQM does not, however, satisfy the broadest possible definition of rationality. The traditional neoclassical agent endowed with complete and transitive psychological preferences will never be led to a worse outcome no matter how the agent's current choices are added to future choice sets. Status quo maintainers on the other hand can end up with dominated options in choice environments where they are not allowed to retain their selection from the preceding round. To see an example, suppose our agent with monotone yet incomplete preferences is offered an initial-round choice from $\{x, w\}$, where $w$ vector dominates $y$ but has less of good 1 than $x$ or $z$. The agent then chooses from $\{y, z\}$ if a second round occurs. Finally, if a third round occurs, the agent must choose between the bundles selected in the first two rounds. A status quo maintainer might choose $x$ in the first round and $y$ in the second, but the agent would necessarily end up better off in any third round if he or she were instead to choose $w$ and $z$ in the first two rounds. More generally (Theorem 6), status quo maintenance can never be outcome-rational in all choice environments.

One might then speculate that in sufficiently sophisticated environments the completeand-transitive neoclassical agent enjoys a rationality edge over agents who sometimes choose intransitively. This speculation turns out not to be true. The main result, Theorem 4, shows that any agent with transitive but incomplete psychological can always 
display intransitivities of choice but not at the cost of outcome irrationality. Thus there is no single definition of preference for which rational agents must satisfy both completeness and transitivity.

\section{Psychological preferences and transitivity}

We consider why self-interested agents should have psychological preference relations that satisfy transitivity. In this section and the next, preferences are defined over a consumption set $X \subset \mathbb{R}_{+}^{n}$. To avoid some minor boundary issues, we assume that $X$ is open.

Psychological preferences are represented by a binary relation $\succcurlyeq$ on $X$. We interpret $x \succcurlyeq y$ to mean that the agent believes that he or she is at least as well off with $x$ as with $y$.

We use the following standard notation. Given the relation $\succcurlyeq$, strict preference $\succ$ is defined by $x \succ y$ if $x \succcurlyeq y$ and not $y \succcurlyeq x$ and indifference $\sim$ by $x \sim y$ if $x \succcurlyeq y$ and $y \succcurlyeq x$. The relation $\succcurlyeq$ is complete if, for all $x, y \in X$, either $x \succcurlyeq y$ or $y \succcurlyeq x$, transitive if, for all $x, y, z \in X, x \succcurlyeq y$ and $y \succcurlyeq z$ imply $x \succcurlyeq z$, and intransitive if not transitive.

Let $x \perp y$ mean not $x \succcurlyeq y$ and not $y \succcurlyeq x$, that is, $\succcurlyeq$ does not rank bundles $x$ and $y$.

When psychological preferences are not complete, a violation of transitivity can occur when merely $x \succcurlyeq y, y \succcurlyeq z$, and $x \perp z$ obtain. But the potential irrationality of intransitivity lies in simultaneously having the preferences $x \succcurlyeq y, y \succcurlyeq z$, and $z \succ x$. To pinpoint this property, define $\succcurlyeq$ to be behaviorally transitive if there do not exist $x, y, z \in X$ such that $x \succcurlyeq y, y \succcurlyeq z$, and $z \succ x$. If $\succcurlyeq$ is complete, then $\succcurlyeq$ is transitive if and only if it is behaviorally transitive.

Given our welfare interpretation of preference, a rational agent must always choose $x$ over $y$ when $x \succ y$ and will see no harm in exchanging $y$ for $x$ when $x \succcurlyeq y$. A rational agent also should not make sequences of decisions that lead to strictly dispreferred outcomes. Anticipating the rationality condition for extended choice functions in Section 5, we call $\succcurlyeq$ outcome-rational if and only if there does not exist a set of alternatives $\left\{x^{1}, \ldots, x^{m}\right\}$ such that $x^{i+1} \succcurlyeq x^{i}$, for $i=1, \ldots, m-1$, and $x^{1} \succ x^{m}$.

To see the appeal of outcome rationality, think of the agent as endowed with an initial bundle $x^{1}$ and then offered the chance to exchange $x^{1}$ for $x^{2}$. If the agent accepts the offer, he or she may end up consuming $x^{2}$ or may receive a new offer to exchange $x^{2}$ for some $x^{3}$, and so on. To ensure that the agent has no incentive to trade counterpreferentially, we must suppose that the offer of any bundle is independent of the agent's past choices, and to ensure that the agent actually cares about each decision, the agent must not know when the sequence of offers will come to an end. When the offers do end, the agent consumes the bundle he or she is currently holding. ${ }^{2}$ With these conditions in place, the link between

\footnotetext{
2 We also need a state-independence condition that $\succcurlyeq$ is unaffected by when the sequence of offers comes to an end. State independence is very mild, but it remains a substantive restriction on preferences. An empirically verifiable preference theory has little choice on this point however; without state independence, one cannot sample multiple elements of a single preference relation over $X$ and any pattern of behavior becomes rationalizable. To define state independence formally, let the state space be $\mathbb{N}$, where $n \in \mathbb{N}$ specifies the date when offers cease. The agent then has preferences $\succcurlyeq^{*}$ over $X \times \mathbb{N}$ and state independence is a joint restriction on $\succcurlyeq^{*}$ and $\succcurlyeq$ : for each $n \in \mathbb{N}$ the projection of $\succcurlyeq^{*}$ onto $X \times\{n\}$ must induce the relation $\succcurlyeq$.
} 
outcome rationality and self-interest is clear: agents who violate outcome rationality may end up with a final consumption bundle $x^{m}$ that is strictly worse than the bundle they began with.

Since a failure of behavioral transitivity means there are alternatives $x, y$, and $z$ such that $x \succcurlyeq y, y \succcurlyeq z$, and $z \succ x$, outcome rationality evidently provides a justification for behavioral transitivity. But this simple argument has a limitation: since an outside observer might not know if an agent has sequentially traded to a dispreferred outcome, one cannot verify through observations of choice whether an agent satisfies outcome rationality.

The money pump argument avoids this drawback by using an external test of an agent's welfare. ${ }^{3}$ The classical money pump considers an agent with a preference cycle over some triple of items, e.g., $x \succ y, y \succ z, z \succ x$, and who is endowed with one of the choices, say $y$. An outsider can then extract money from the agent by selling the agent the chance to switch to $x$. Since $x \succ y$, the agent will pay some sum of money in order to move to $x$. The agent will then pay for similar offers to move to $z$ and back to $y$, thus ending up with the original option, but with less money. In some versions, the pump is iterated and money is extracted repeatedly. To state an explicit theorem that intransitivity necessarily exposes an agent to a money pump, some adjustments to the classical story are needed: the argument should to apply to preferences over goods rather than money, and the agent should be exposed to manipulation whenever $\succcurlyeq$ violates transitivity, not just when $\succcurlyeq$ has strict preference cycles. Our treatment indicates that a mild continuity restriction on preferences is needed for a money-pump result (and is indeed implicit in the story).

The irrationality of being money-pumped lies in the willing acceptance of a sequence of trades that leads to an obviously inferior outcome, a loss of money. To avoid mentioning money, we can instead consider $\succcurlyeq$ to be irrational if $\succcurlyeq$ can generate choice sequences that lead to a loss of all goods.

Definition 1. A preference relation $\succcurlyeq$ is outcome-monotonic if and only if whenever $\left\{x^{1}, \ldots, x^{m}\right\}$ satisfies $x^{m} \succcurlyeq \cdots \succcurlyeq x^{1}$ then not $x^{1} \gg x^{m} \cdot 4$

Outcome rationality and outcome monotonicity impose similar rationality restrictions; the former precludes choice sequences that lead to dispreferred outcomes and the latter precludes sequences that lead to decreases of all goods.

Let $\succcurlyeq$ be lower continuous if and only if, for any $x \in X,\{y: y \succ x\}$ is open. Given the welfare interpretation of $\succcurlyeq$, lower continuity has a traditional rationale: insofar as an agent's welfare stems from a partly physiological sense of satisfaction, it is reasonable to think that welfare will vary continuously with consumption.

Theorem 1. If $\succcurlyeq$ is outcome-monotonic and lower continuous, then $\succcurlyeq$ is behaviorally transitive.

\footnotetext{
3 The money pump originates in Davidson et al. (1955). See Raiffa (1968), Fishburn (1988), Anand (1993), and Cubitt and Sugden (2001) for pro and con views.

4 Letting subscripts denote coordinates, we use the notation: $x \geqslant y \Leftrightarrow x_{i} \geqslant y_{i}$, all $i ; x>y \Leftrightarrow x \geqslant y, x \neq y$; and $x \gg y \Leftrightarrow x_{i}>y_{i}$, all $i$.
} 
Proof. If $\succcurlyeq$ is not behaviorally transitive, there exist $x, y, z \in X$ such that $x \succcurlyeq y, y \succcurlyeq z$, and $z \succ x$. By lower continuity, there exists a $\varepsilon>0$ such that $(1-\varepsilon) z \succ x$, and so the sequence $z, y, x,(1-\varepsilon) z$ is increasing according to $\succcurlyeq$. Since $z \gg(1-\varepsilon) z$, $\succcurlyeq$ violates outcome monotonicity.

Outcome monotonicity places restrictions on chains of weak preference decisions and thus presupposes that agents are willing to switch between indifferent alternatives; and given the welfare interpretation of preference, agents ought to be willing to make such exchanges. But if $\succcurlyeq$ satisfies full continuity and a form of local nonsatiation, and if outcome monotonicity were weakened to require only that chains of strictly preferred options do not lead to endowment reductions, then $\succcurlyeq$ must also satisfy behavioral transitivity. We omit the details, which are routine.

\section{Choice functions and completeness}

By far the most common rationale for completeness argues that since agents would rather consume something than nothing, they will choose some subset of items from any set of possibilities, and these choices can be said to be preferred to the unselected items. The choice function literature, which takes choice rather than preference as primitive, formalizes this position. Given a nonempty set of options $S$, a choice function $C$ identifies a nonempty subset of $S, C(S)$, interpreted as the options that the agent designates as his or her choices when offered $S$. We retain the consumption set $X$, an open subset of $\mathbb{R}^{n}$, from the previous section and assume that the domain of $C$ consists of all finite subsets of $X$. We follow the standard convention that agents can designate multiple options as permissible choices: $C(S)$ need not be a singleton. The multiple-option possibility occurs when the agent specifies more than one bundle as acceptable. Such an action would be observable, but means that some other procedure (e.g., randomization, a decision by the offerer) must determine the exact bundle in $C(S)$ that is in the end consumed.

Preference relations can be derived from choice functions in several ways. We use the most common definition of the (weak) revealed preference relation $R$, namely $x R y$ if and only if there exists a finite $S \subset X$ such that $x \in C(S)$ and $y \in S .{ }^{5}$ Strict revealed preference $P$ is defined by $x P y$ if and only if $x R y$ and not $y R x$.

We investigate $R$ rather than some other definition of preference because $R$ is necessarily complete and there is a seemingly plausible case for the transitivity of $R$-and our aim is to see if there is a single definition of preference for which a rational agent will satisfy both axioms. ${ }^{6}$

\footnotetext{
5 See, e.g., Mas-Colell et al. (1995). The Arrow (1959) definition, perhaps the next most common for weak revealed preference, is $x R^{\prime} y$ if and only if $x \in C(\{x, y\})$. The proof of Theorem 3 below is easily adjusted to apply to $R^{\prime}$ rather than $R$.

6 See Nehring (1997) and Eliaz and Ok (2002) for analyses of when choice functions may be rationalized by incomplete binary relations. Danan (2001), which calls psychological preferences cognitive, considers the related problem of when a revealed preference relation can be rationalized by an incomplete cognitive preference relation.
} 
The completeness of $R$ follows from the fact that $C$ specifies nonempty subsets; in particular $C(\{x, y\})$ is nonempty. But must a self-interested agent have a transitive $R$ ? An agent with an incomplete but transitive preference relation $\succcurlyeq$ can, if desired, select a choice function whose revealed preference relation both agrees with the orderings that are contained in $\succcurlyeq$ and is transitive. ${ }^{7}$ But must a rational agent utilize such a choice function? The most famous theorem of the choice function literature addresses this point. The choice function $C$ satisfies the weak axiom if and only if $x R y$ implies that there does not exist a finite $S$ such that $y \in C(S), x \in S$, and $x \notin C(S)$.

Theorem 2. If the choice function $C$ satisfies the weak axiom, the revealed preference relation $R$ is transitive.

Proof. Arrow (1959).

If choice is determined by a fixed and complete set of welfare judgments, the weak axiom is plausible: an agent who chooses $x$ when $y$ is available must think that $x$ delivers as much welfare as $y$, and should persist in that judgment when faced with another choice set that includes $x$ and $y$. But if choice does not always reveal a psychological preferencebecause psychological preferences are incomplete - the weak axiom is hardly compelling: if $x$ and $y$ are psychologically unranked, there is nothing contradictory in choosing $x$ over $y$ on one occasion, and refusing to do so on another occasion. Only counterpreferential choice is prima facie irrational; see Sen (1973) on this point.

A more promising rationale for the transitivity of revealed preferences is to extend our earlier result, Theorem 1, on the irrationality of intransitive psychological preferences. Indeed, the logic of outcome monotonicity applies more straightforwardly to revealed preferences than to psychological preferences: first, since $R$ is complete, transitivity and behavioral transitivity are equivalent, and second, since agents' willingness to choose defines revealed preference, the possibility of agents refusing to switch to options classified as indifferent does not arise.

\section{Definition 2. A choice function $C$ is}

- outcome-monotonic if and only if whenever there exist $x^{0}, \ldots, x^{m}$ and $S^{1}, \ldots, S^{m}$ such that $x^{k-1} \in S^{k}, x^{k} \in C\left(S^{k}\right), k=1, \ldots, m$, then not $x^{0} \gg x^{m}$,

- lower continuous if and only if for all $x,\{y: y P x\}$ is open.

The lower continuity of choice functions is justified as follows. If, for all choice sets $S$ that contain both $x$ and $y$, an agent never chooses $y$ and sometimes chooses $x$, it is reasonable to infer that the agent believes him or herself to be strictly psychologically better

\footnotetext{
7 To see why, note that if $\succcurlyeq$ is transitive then it induces an irreflexive and transitive ordering of the equivalence classes of $\succcurlyeq$. Hence Szpilrajn's theorem (see the proof of Theorem 4) implies that there exists a linear ordering $\succ^{*}$ of the equivalence classes of $\succcurlyeq$ such that, letting $I(x)$ denote $\{w: w \sim x\}, x \succ y$ implies $I(x) \succ^{*} I(y)$ for all $x$ and $y$. Setting $C(S)=\left\{x \in X: \nexists y \in S\right.$ with $\left.I(y) \succ^{*} I(x)\right\}$, the resulting $R$ is complete and transitive, $x \succcurlyeq y$ implies $x R y$, and $x>y$ implies $x P y$.
} 
off with $x$ than with $y .{ }^{8}$ Following the standard rationale for the continuity of psychological preferences, the agent should also regard any bundle $w$ sufficiently near to $x$ as strictly preferable to $y$. The agent would therefore never choose $y$ when $w$ is available, which means that the agent's choice function is lower continuous. Some psychological content thus underlies the lower continuity of choice functions, but the content is minimal.

Theorem 3. If $C$ is outcome-monotonic and lower continuous, then $R$ is transitive.

Proof. With the appropriate changes in notation, the proof proceeds as in Theorem 1. Since $R$ is complete, intransitivity implies the existence of $x, y, z$ such that $x R y, y R z$, $z P x$. By the definition of $R$, there exists a $S^{1}$ such that $y \in C\left(S^{1}\right)$ and $z \in S^{1}$, and a $S^{2}$ such that $x \in C\left(S^{2}\right)$ and $y \in S^{2}$. Since $z P x$, lower continuity implies there is a $\varepsilon>0$ such that, setting $S^{3}=\{x,(1-\varepsilon) z\},(1-\varepsilon) z \in C\left(S^{3}\right)$. Hence, we have a violation of outcome monotonicity.

Theorem 3 seems to offer a convincing case: agents with intransitive $R$ s are exposed to manipulations that lead to a loss of all goods. Self-interest therefore appears to dictate that agents behave as if they possess a preference relation that is both complete and transitive.

\section{Extended choice functions}

We now argue that the apparent drawback of intransitive revealed preference is an artifact of requiring that choice functions specify a single set of choices for each set of options, that is, a single set $C(S)$ for each $S$. To that end, we now define choice functions on sequences of choice sets, thereby allowing choice to be conditioned on past decisions. Our main result is that if psychological preferences are incomplete and transitive, then outcomerational choices can generate intransitive revealed preferences. Hence no argument similar to Theorem 3 can show that intransitive choosers can always be manipulated successfully or otherwise induced to trade away their endowment. Agents with intransitive revealed preferences can therefore share the same rationality advantages as agents with complete and transitive psychological preferences.

Our setting will expose agents to a wide array of decision-making complexities, both simple money pumps, where agents can always retain their current choices into the succeeding period, and to situations where current choices can affect future choice sets only by indirect routes. We must admit the more complex cases: otherwise we could not address whether outcome-rational and intransitive choice behavior can persist in the precisely the same settings that complete and transitive preferences satisfy outcome rationality.

We make several supplementary assumptions, for example, that intransitivities are not trivial and can be observed along a single choice sequence. The complexity of the choice framework and our supplementary assumptions make our intransitivity result stronger:

\footnotetext{
8 Because of the possibility of incomplete psychological preferences, in cases where an agent sometimes chooses $y$ and sometimes chooses $x$ from sets containing both $x$ and $y$ it is not reasonable to infer that the agent is even weakly better off with either $x$ or with $y$.
} 
without them, the outcome rationality of intransitive choice would still obtain, and be easier to establish.

Before proceeding to the model, we consider the reasons for letting agents condition their current decisions on the choice sets faced in the past. The supposed advantage of choice functions is that choice, unlike psychological preference, can be directly observed. But to observe multiple samples from a choice function, the agent must choose repeatedly; otherwise, an observer would know only the choices from a single $S$, not the mapping $C$. By defining choice functions on sequences of choice sets, we make the sequence of decisions explicit. In addition, as Theorem 3 makes clear, the disadvantages of intransitivity arise only when agents choose repeatedly; so, to assess the rationality of transitivity, we must consider a sequential model. But when choice is sequential, agents can condition current decisions on past choice sets or past decisions. Status quo maintenance (SQM) holding to one's current option until offered a superior alternative-is a prominent example of such a rule. To see why, suppose a status quo maintainer does not psychologically rank bundles $x$ and $y$ but strictly prefers either $x$ or $y$ to $w$. If the agent first faces the choice set $\{x, w\}$ and then the selection from this round versus $y$, the agent will choose $x$ in both rounds. But if the agent first faces $\{y, w\}$ and then the first-round selection versus $x$, the agent will choose $y$ in both rounds. Thus, the second-round selection from $\{x, y\}$ depends on the first-round choice set. So, to consider the outcome rationality of SQM and other decision rules, we must permit history-dependent choice.

The model considers an agent who, at each positive integer $t$, faces a finite, nonempty choice set $S^{t} \subset X$, where $X$ is an arbitrary consumption set with at least three elements. At each $t$, the agent identifies a nonempty subset of $S^{t}$ as his or her set of selections. As in Section 3, each $S^{t}$ contains commodity bundles to be consumed at some date following the agent's choice sequence, and the agent does not know at each $t$ if that date's decisions will determine his or her ultimate consumption, or whether instead a further set of choices will be offered. Let $\Sigma$ denote the set of sequences of finite choice sets: $\sigma \in \Sigma$ if and only if $\sigma=S^{1} \times \cdots \times S^{t} \times \cdots$, where each $S^{t} \subset X$ is nonempty and finite. ${ }^{9}$ The agent's welfare is gauged by a psychological preference relation $\succcurlyeq$ defined over $X{ }^{10}$

The agent's behavior is represented by an extended choice function $\gamma: \Sigma \rightarrow \Sigma$ where, for each $\sigma \in \Sigma, \gamma(\sigma) \subset \sigma$. We define $\gamma$ on the entire space $\Sigma$ to ensure that the agent faces a broad array of potential choice hazards that includes but is not restricted to the money pump.

Let $\gamma^{t}(\sigma) \subset S^{t}$ denote the agent's choices at $t$, and given $\sigma$, let $\sigma^{t}$ denote $S^{1} \times \cdots \times S^{t}$. We require extended choice functions to satisfy the following measurability condition at each $t$ : if $\sigma$ and $\hat{\sigma}$ are such that $\sigma^{t}=\hat{\sigma}^{t}$, then $\gamma^{t}(\sigma)=\gamma^{t}(\hat{\sigma})$. Agents, that is, cannot condition their current choices on the choice sets they encounter in the future. Given $\gamma$,

\footnotetext{
9 The absence of a fixed terminal date is unavoidable: since terminal-date decisions are not subject to all of the drawbacks of sequential choice, a convincing demonstration that rational agents can choose intransitively should not hinge on those decisions.

10 As in Section 3, to take multiple samples from a single preference relation over $X$, we need in the background a state-independence assumption that the agent's preferences over $X$ are unaffected by the resolution of the uncertainty as to when the sequence of choices ends and how choices at $t$ affect $S^{\tau}$ for $\tau>t$.
} 
redefine the revealed preference relation $R$ by $x R y$ if and only if there exists some $t$ and $\sigma$ such that $x \in \gamma^{t}(\sigma)$ and $y \in S^{t}$. Define $P$ by $x P y$ if and only if $x R y$ and not $y R x$.

We now define and explain outcome rationality for extended choice functions. Given an extended choice function $\gamma$, period $t$ is linked to period $\tau$ at $\sigma$ if $\tau<t$ and one of the elements selected during period $\tau$ is in the choice set of period $t: S^{t} \cap \gamma^{\tau}(\sigma) \neq \varnothing$. Loosely speaking, an extended choice function is outcome-rational if there does not exist a finite sequence of periods, with each period linked to its immediate predecessor, such that the agent in the final period chooses a bundle that is worse than any $x$ in the choice set of the initial period. If there were such a sequence, then the agent, had he or she chosen only $x$ in each period of the sequence, would have ended up with a superior outcome.

Definition 3. An extended choice function $\gamma$ is outcome-rational if and only if whenever there is a $\sigma$, a set of time periods $\left\{t_{1}, t_{2}, \ldots, t_{m}\right\}, x^{1} \in S^{t_{1}}$, and $x^{m} \in \gamma^{t_{m}}(\sigma)$ such that $t_{j}$ is linked to $t_{j-1}$ at $\sigma$ for $2 \leqslant j \leqslant m$, then not $x^{1} \succ x^{m}$.

Since $m$ can equal 1 , an outcome-rational $\gamma$ cannot, at any $t$, specify a $\gamma^{t}$ that contains a bundle dominated by another element of $S^{t}$.

Definition 3 considers arbitrary linkages between the agent's current choices and future choice sets to ensure that outcome-rational agents do not end up with inferior final consumption bundles in exactly the same environments in which choice guided by complete and transitive preferences does not lead to inferior final consumption. In the simple sequential offers considered in Section 3, the agent chose one bundle at each date with the subsequent choice set consisting of that bundle and one alternative bundle. But if an agent's choices are guided by complete and transitive preferences, current choices could be added to any number of future choice sets and still the agent would not be better off if he or she were to choose counterpreferentially. So we have stated outcome rationality to cover cases where an agent's current choices or any subset of those choices are added to any future choice set. We allow only current choices themselves to be added to future choice sets since otherwise (if a current choice could lead other bundles to enter a future choice set) even complete-and-transitive agents would sometimes have a disincentive to choose the most preferred bundle currently on offer.

We do not need to specify this causal connection between current choices and future choice sets for our formal result; it matters only for the interpretation of outcome rationality as an axiom of self-interest. The precise relationship between $\gamma$ and $\sigma$ needed for this interpretation is as follows. Each $\sigma$ is constructed from a nonempty set of base options $\bar{\sigma}=\bar{S}^{1} \times \cdots \times \bar{S}^{t} \times \cdots$, where each $\bar{S}^{i}$ is finite. The set the agent actually chooses from at time $t, S^{t}$, equals the union of $\bar{S}^{t}$ and some (possibly empty) subset of the options chosen during previous periods. For the initial period, $S^{1}=\bar{S}^{1}$. At each period $i$, therefore, the only influence of $\gamma^{i}$ on $\sigma$ is that $S^{t}, t>i$, may include some of the elements of $\gamma^{i}$. Let $s_{i}^{t}\left(\gamma^{i}\right)$ denote the (possibly empty) subset of $\gamma^{i}$ that is appended to $\bar{S}^{t}$. Thus, each $S^{t}$, $t \geqslant 2$, equals the union of $\bar{S}^{t}$ and $\left\{s_{i}^{t}\left(\gamma^{i}\right)\right\}_{i=1}^{t-1}$. We assume for all $t$, all $i<t$, and all $\bar{\sigma}$, that if $s_{i}^{t}\left(\gamma^{i}\right)$ is empty for some $\gamma^{i}$ then it is empty for all $\gamma^{i}$. Thus the agent's actions do not affect whether or not some of the period $i$ choices are appended to $\bar{S}^{t}$, which eliminates any advantage to choosing counterpreferentially. Finally we must suppose that the agent believes for all $t$ and $i<t$ that each function $s_{i}^{t}$ that meets the above conditions could be 
the true $s_{i}^{t}$, thus ensuring that the agent thinks it possible that any period $i$ choice could enter any future choice set.

If $\succcurlyeq$ were not transitive, then the arguments of Section 3 imply that no $\gamma$ could be outcome-rational. Our program is to show that if $\succcurlyeq$ is incomplete and transitive, then there will exist outcome-rational $\gamma$ s such that $R$ is intransitive. Before stating the result, we introduce two final restrictions to ensure that the intransitivity we demonstrate is not vacuous.

The first restriction addresses the potential for trivial intransitivities of choice in which agents always select only one of a pair of bundles that are indifferent or unranked. To see the difficulty, let $x, y$, and $z$ all be indifferent: $x \sim y \sim z$. An outcome-rational agent might refuse to choose $x$ whenever $z$ is available, which could lead to the intransitive revealed preferences $x R y R z P x$. Thus, even an outcome-rational agent with complete and transitive psychological preferences could fail to have transitive revealed preferences. A similar point arises with unranked bundles. For example, recalling that $a \perp b$ means not $a \succcurlyeq b$ and not $b \succcurlyeq a$, the intransitivity $x R y R z P x$ is consistent with outcome rationality if $z \perp x \sim y \perp z$. In both of these examples, the agent suffers no harm with a choice sequence that leads from an initial $z$ to a final outcome $x$, and so a self-interested agent can persist in this behavior. The intransitivities are farfetched, however, since the agent has no incentive to avoid an indifferent or unranked bundle at each and every choice set.

We rule the above intransitivities out with the following requirement.

Definition 4. The extended choice function $\gamma$ obeys $\succcurlyeq$ if and only if for all $x, y \in X$ and all $\sigma \in \Sigma$, (1) $x \sim y, y \in \gamma^{t}(\sigma), x \in S^{t} \Rightarrow x \in \gamma^{t}(\sigma)$, and (2) $x \perp y \Rightarrow x R y .^{11}$

Since an extended choice function $\gamma$ determines the revealed preference relation $R,(2)$ is indirectly a restriction on $\gamma$. Condition (1) says that if the agent chooses a bundle $y$ and $x$ is indifferent to $y$ and is also an available choice, then $x$ is chosen too, while (2) says that if $x$ and $y$ are unranked then the agent sometimes chooses $x$ when both $x$ and $y$ are available. An outcome-rational $\gamma$ that obeys $\succcurlyeq$ must therefore "pick up" all undominated bundles. If $x$ is undominated by some $y$ (that is, not $y \succ x$ ), then $x \succ y$, or $x \sim y$, or $x \perp y$. In the first case, an outcome-rational $\gamma$ must sometimes select $x$ when $y$ is available, e.g., when $S^{t}=\{x, y\}$. When $x \sim y$ then (1) implies that $\gamma^{t}(\sigma)$ contains $x$ whenever $\gamma^{t}(\sigma)$ contains $y$, and vice versa. And when $x \perp y$ then (2) implies there is some $t$ and $\sigma$ such that $x \in \gamma^{t}(\sigma)$ and $y \in S^{t}$.

For the neoclassical agent with a complete and transitive $\succcurlyeq$, an outcome-rational $\gamma$ that obeys $\succcurlyeq$ must generate a transitive $R$. In this case, in fact, an outcome-rational $\gamma$ obeys $\succcurlyeq$ if and only if each $\gamma^{t}$ equals the set of undominated bundles in $S^{t}$. It is the possibility of an incomplete $\succcurlyeq$ that opens the door to an intransitive $R$.

The second restriction will ensure that there is an intransitivity of choice along a single sequence $\sigma$ of choice sets. The potential danger of an intransitive $R$ is that a choice sequence can lead to a dispreferred outcome (or an endowment reduction). Consequently, an intransitivity $x \operatorname{RyR} P x$ such that $x$ is chosen over $y$ only at a $\sigma$ where $y$ is never chosen

\footnotetext{
11 If $\gamma$ does not obey $\succcurlyeq$, there is no reason for $z P x$ to be "open": $z P x$ would not imply $z \succ x$ and so bundles near $z$ need not always be chosen over $x$. Hence the outcome monotonicity arguments of Sections 3 and 4 could not be used to establish transitivity.
} 
over $z$ would not be so remarkable - an agent could, after all, use different complete and transitive $R \mathrm{~s}$ at different $\sigma \mathrm{s}$. It is an intransitivity along a single $\sigma$ that conceivably could lead to a failure of outcome rationality. In addition, an empirically verifiable intransitivity must occur at a single $\sigma$ - that is the most an outside observer can see.

Definition 5. The extended choice function $\gamma$ is verifiably intransitive if and only if there exists a $\sigma$ and a triple $x, y, z$ such that $x R y R z P x$ and (1) $x \in \gamma^{t}(\sigma)$ and $y \in S^{t}$ for some $t$, (2) $y \in \gamma^{t^{\prime}}(\sigma)$ and $z \in S^{t^{\prime}}$ for some $t^{\prime}$, and (3) $z \in \gamma^{t^{\prime}}(\sigma)$ and $x \in S^{\hat{t}}$ for some $\hat{t}$. ${ }^{12}$

Let $\succcurlyeq$ be nontrivially incomplete if and only if there exist $x, y$, and $z$ such that $x \perp y$, $y \perp z$, and $z \succ x$.

Theorem 4. If $\succcurlyeq$ is transitive and nontrivially incomplete, there exist outcome-rational extended choice functions that obey $\succcurlyeq$ and are verifiably intransitive.

Although the proof, in Appendix A, is lengthy, the key idea is simple. Verifiable intransitivity requires there to be a revealed preference for $x$ over $y$ at some $S^{i}$ and a revealed preference for $y$ over $z$ at some $S^{k}$ (where $x \perp y, y \perp z$, and $z \succ x$ ). In periods following $S^{i}$ and $S^{k}$, outcome rationality requires that $y$ is chosen over $x$ (otherwise $x$ would be indirectly linked via a chain of time periods to the earlier choice of $z$ ). But outcome rationality also requires that, following $S^{i}$ and $S^{k}, x$ is chosen over any $w \prec y$ (otherwise $w$ would be indirectly linked to $y$ ). Showing that a $\gamma$ can comply with these restrictions and simultaneously satisfy our other stipulations forms the heart of the proof.

Since $\succcurlyeq$ may satisfy a monotonicity assumption, Theorem 4 implies that an agent with monotone preferences who uses an outcome-rational $\gamma$ will be immune to all endowmentreducing manipulations. Since $\gamma$ is defined on all of $\Sigma$, these manipulations include but are not restricted to the classical money pump. Indeed, we defined $\gamma$ on $\Sigma$ to ensure that the agent faces a large variety of potential manipulations. To recast Theorem 4 in terms of endowment reductions, define an extended choice function $\gamma$ to be outcome-monotonic if and only if whenever there is a $\sigma$, a finite set of time periods $\left\{t_{1}, t_{2}, \ldots, t_{m}\right\}, x^{1} \in S^{t_{1}}$, and $x^{m} \in \gamma^{t_{m}}(\sigma)$ such that $t_{j}$ is linked to $t_{j-1}$ for $j=2, \ldots, m$ then not $x^{1}>x^{m}$. ${ }^{13}$ Define a preference relation $\succcurlyeq$ to be increasing if and only if $x>y$ implies $x>y$.

Corollary. If $\succcurlyeq$ is increasing, transitive and nontrivially incomplete, there exist extended choice functions that obey $\succcurlyeq$ and that are outcome-monotonic and verifiably intransitive.

A final interpretive comment will be helpful. We justified outcome rationality and measurability by assuming that an agent does not know if his or her current choices will be

\footnotetext{
12 A fact such as $z P x$ can be confirmed empirically only if all $(\sigma, \gamma)$ such that some $S^{t} \supset\{x, z\}$ are observed. Theorem 4 does not exploit this observational limitation, however, since it establishes the intransitivity of $R$, which is defined using all choice sequences not just one.

13 Since we want the rationality properties of extended choice functions that display intransitivity to be as strong as possible, we define outcome monotonicity for extended choice functions to be more demanding than outcome monotonicity for psychological preference relations: an agent with an outcome-monotonic extended choice function cannot end up with a bundle $x^{m}$ that is even weakly vector dominated by $x^{1}$.
} 
carried forward into future choice sets. This is the easiest rationale, but we could instead suppose that the agent at time 1 knows $\bar{\sigma}$, knows the $s_{i}^{t}$ functions, knows the terminal date, say $T$, of the choice sequence, and knows which element of $\gamma^{T}$ will be the agent's actual consumption bundle. The agent would then face no uncertainty. We can understand measurability in this scenario as a simplicity requirement: agents do not take the effort to condition their current choices on future choice sets. Theorem 4 says that outcome-rational $\gamma \mathrm{s}$, which by definition satisfy measurability, can generate intransitive choices; if the measurability requirement on the $\gamma$ were dropped, the same conclusion would continue to hold. Of course, our result is stronger with measurability in place.

\section{Status quo maintenance: rational intransitivity in simple choice environments}

We turn to the prominent special case where choice sets in each period are linked to their immediate predecessors, thus allowing agents always to reserve an option from the previous period. Status quo maintenance then illustrates our main point that outcome rationality and intransitive choice are mutually consistent when psychological preferences are incomplete. Behavioral economists have established that agents frequently exhibit SQM or an endowment effect: decision-makers are more likely to choose whatever bundle they happen to be initially endowed with, thus apparently showing that agents' preferences change as a function of the choice sets they face (see, e.g., Thaler, 1980; Samuelson and Zeckhauser, 1988; Knetsch, 1989). Incomplete preferences offer a competing explanation of SQM that allows the phenomenon to arise from a single unchanging preference relation (Mandler, 1998, 2004). By assuming that preferences do not change, we can see that SQM can be outcome rational and hence that self-interest need not weed it out.

We say that the choice environment is simple if and only if $(\sigma, \gamma)$ is such that, for $t \geqslant 2$, period $t$ is linked to period $t-1$. If the choice environment is not simple, it is complex. Experiments in economics usually involve simple choice environments: agents are endowed with bundles of goods and asked whether they would like to switch to a new bundle.

Definition 6. An extended choice function $\gamma$ is outcome-rational on simple choice environments if and only if whenever the choice environment is simple and there are $\left\{t_{1}, t_{2}, \ldots, t_{m}\right\}, x^{1} \in S^{t_{1}}$, and $x^{m} \in \gamma^{t_{m}}(\sigma)$ such that $t_{j}$ is linked to $t_{j-1}$ at $\sigma$ for $2 \leqslant j \leqslant m$, then not $x^{1} \succ x^{m}$.

In line with our interpretation of the sequential choice model in Section 5, a simple choice environment can arise in the following way. At any $t$, the agent must choose an element of $S^{t}$. In period $1, S^{1}=\bar{S}^{1}$, but subsequently $S^{t}$ is the union of $\bar{S}^{t}$ and at least one of the options selected at $t-1$. That is, for $t \geqslant 2, S^{t}=\bar{S}^{t} \cup X^{t-1}$ for some nonempty $X^{t-1} \subset \gamma^{t-1}$. Since the agent's effect on next period's choice set is only to augment it with items chosen in the current period, the agent has no incentive to choose counterpreferentially. 
Definition 7. An extended choice function $\gamma$ maintains the status quo if and only if (1) at each $t$ and $\sigma, x \in \gamma^{t}(\sigma)$ implies there does not exist a $x^{\prime} \in S^{t}$ such that $x^{\prime} \succ x$, and (2) if $t \geqslant 2$ and $S^{t} \cap \gamma^{t-1}(\sigma) \neq \varnothing$, then $x \in \gamma^{t}$ implies $x \succcurlyeq \hat{x}$ for some $\hat{x} \in S^{t}(\sigma) \cap \gamma^{t-1}$.

That is, the agent does not choose dominated bundles and if a period is linked to the previous period, then any currently chosen bundle must be at least as good as some $\hat{x}$ that was chosen in the previous period and passed on to the current choice set. Unlike the traditional presentation of SQM, the agent's preferences do not change as a function of his or her choice set; the same relation rules throughout.

To check that maintaining the status quo can generate intransitive choices, consider this simple example. Suppose $\succcurlyeq$ is transitive and nontrivially incomplete, i.e., there exist $x, y$, and $z$ such that $x \perp y, y \perp z$, and $z \succ x$. Let $(\sigma, \gamma)$ be such that $S^{1}=\{x, y\}, S^{2}=\{x, y, z\}$, $\gamma^{1}(\sigma)=\{x, y\}$, and $\gamma^{2}(\sigma)=\{y, z\}$. Plainly, $\gamma^{1}(\sigma)$ and $\gamma^{2}(\sigma)$ are consistent with $\gamma$ maintaining the status quo. (The proof of Theorem 5 below implies that $\gamma$ on the remainder of its domain may be set so that $\gamma$ maintains the status quo at every $t$.) We have $x R y$ and $y R z$. But since a $\gamma$ that maintains the status quo cannot choose dominated bundles at any $S^{t}$, we must have $z P x$; so $R$ is intransitive. Observe that $\gamma$ obeys $\succcurlyeq$ on $\{x, y, z\}$ since $y R x, x R y$, $y R z$, and $z R y$, and so the intransitivity is not of the trivial variety mentioned in Section 5 .

Theorem 5. If $\succcurlyeq$ is transitive, then $\gamma$ 's that maintain the status quo exist and any $\gamma$ that maintains the status quo is outcome-rational on simple choice environments.

Proof. Existence. If $t \geqslant 2$ and there is some $x^{\prime}$ in $S^{t} \cap \gamma^{t-1}$, let $\gamma^{t}=\{v\}$, where $v$ is an undominated element of $A=\left\{w \in S^{t}: w \succcurlyeq x^{\prime}\right\}$; the transitivity of $\succcurlyeq$ and the finiteness of $S^{t}$ imply that such a $v$ exists. Otherwise, let $\gamma^{t}$ consist of an arbitrary undominated element of $S^{t}$. In the first case, $\gamma^{t}$ must be undominated by bundles in $S^{t}$ since if there were a $u \in S^{t} \backslash A$ such that $u \succ v$, then by transitivity $u \succcurlyeq x^{\prime}$ and hence $u \in A$, a contradiction.

Outcome rationality. It is sufficient to show that for all $t \geqslant 1$ there do not exist $x$ and $x^{\prime}$ such that $x \in \gamma^{t}, x^{\prime} \in \bigcup_{i=1}^{t} S^{i}$, and $x^{\prime} \succ x$. By (1) of Definition 7, this holds for $t=1$. To show that if the statement holds for $t$ then it holds for $t+1$, suppose $x \in \gamma^{t+1}$ and $x^{\prime} \succ x$. By (2) of Definition 7, $x \succcurlyeq w$ for some $w \in \gamma^{t}$. By transitivity, $x^{\prime} \succ w$. But $x^{\prime} \notin \bigcup_{i=1}^{t} S^{i}$, since by the induction assumption any $w \in \gamma^{t}$ is undominated by any element of $\sigma^{t}$, and $x^{\prime} \notin S^{t+1}$ since by (1) $x$ is undominated by any element of $S^{t}$. Hence $x^{\prime} \notin \bigcup_{i=1}^{t+1} S^{i}$.

As long as the choice environment is simple, the conservatism of status quo maintenance can be relaxed somewhat without requiring agents to perform difficult choice calculations or causing a failure of outcome rationality: at any $t$, agents may choose any or all bundles in $S^{t}$ that are undominated by any element of $\bigcup_{i=1}^{t} S^{i}$ (including bundles in $S^{t}$ that do not dominate any element of $S^{t} \cap \gamma^{t-1}$ ).

If we drop the restriction to simple choice environments-so that agents may no longer be able to reserve options from the preceding period-the outcome rationality of maintaining the status quo then disappears, as Theorem 6 reports. Of course, Theorem 4 implies that other choice functions both exhibit intransitivity of choice and preserve outcome rationality. 
Theorem 6. If $\succcurlyeq$ is nontrivially incomplete, any $\gamma$ that maintains the status quo fails to be outcome-rational.

Proof. Given nontrivial incompleteness, there exist $x, y$, and $z$ such that $x \perp y, y \perp z$, and $z \succ x$. Let $\sigma$ satisfy $S^{1}=\{y\}, S^{2}=\{y, z\}, S^{3}=\{x\}, S^{4}=\{x, y\}$. Any $\gamma$ that maintains the status quo must have $\gamma^{1}(\sigma)=\{y\}, \gamma^{2}(\sigma)=\{y\}, \gamma^{3}(\sigma)=\{x\}, \gamma^{4}(\sigma)=\{x\}$. Since $\gamma^{2}(\sigma) \cap S^{4} \neq \varnothing$, period 4 is linked to period 2. Since in addition $z \in S^{2}, x \in \gamma^{4}$, and $z \succ x$, $\gamma$ is not outcome-rational.

The inability of agents to reserve a bundle from the immediately preceding choice set will not by itself produce a failure of outcome rationality. As the $\sigma$ constructed in the proof makes clear, it is also necessary that a choice set inherit bundles from multiple preceding periods. Specifically, one may show that if, for all $t \geqslant 2$, at most one of the sets $S^{t} \cap \gamma^{\tau}, \tau<t$, is nonempty, then extended choice functions that maintain the status quo are outcome-rational.

\section{Conclusion}

Since rational agents with incomplete psychological preferences can choose intransitively, one cannot argue under either of the standard definitions of preference that rationality entails both completeness and transitivity. When preference is a psychological judgment of well-being, there are persuasive arguments for why preferences should be transitive but not for why they should be complete; when preference is defined as choice, Theorem 4 shows that rational revealed preferences will be complete but not necessarily transitive.

We close with two final comments.

\subsection{Incomplete vs. changing preferences}

Instead of relinquishing the completeness assumption, a more common response to anomalous choice behavior and SQM in particular is to assume that preferences vary as a function of time or an agent's endowment. See for example Tversky and Kahneman (1991), and Kahneman et al. (1990) for support for a similar view. By letting preferences change through time, behavioral models can allow preferences at each date to be complete and transitive. But the behavioral approach presents difficulties.

First, welfare analysis becomes impossible. In particular, since an agent's wellbeing is no longer identified with a single preference relation, the rationality of a choice sequence cannot be assessed by whether the agent is better off at the end of the sequence. Curiously, and in contrast to Section 6 above, one then cannot judge whether self-interest works for or against the persistence of SQM.

Second, time or endowment dependent preferences posit rankings that can never be checked against behavior: for each endowment, only choice vis-à-vis that endowment can be observed. One may postulate that these unobservable preferences are complete and transitive, of course, but since these preferences are irrelevant for decision-making or the agent's welfare, there is no reason why a rational agent should satisfy these assumptions. 
The observability problem may seem instead to be a defect of models of incomplete preferences. Incompleteness implies a distinction between choice and preference (or between what is not chosen and what is dispreferred) and this may appear untestable. This is not the case. As long as an extended choice function $\gamma$ obeys $\succcurlyeq, \gamma$ allows for exact tests. Testing strict preference is straightforward: if $\gamma$ obeys $\succcurlyeq$ and is outcome rational, strict psychological preference obtains if and only if strict revealed preference occurs. To test for indifference between $x$ and $y$, one checks whether $\{x, y\} \subset S^{t}$ and either $x$ or $y$ being an element of $\gamma^{t}$ together imply that both $x$ and $y$ are elements of $\gamma^{t}$. If $\gamma$ obeys $\succcurlyeq$ and $x \sim y$, an agent's choice must satisfy this property. Obeying $\succcurlyeq$ is a plausible requirement, moreover, since if an agent is willing to choose $x$, and $x$ is truly psychologically interchangeable with $y$, then the agent should be willing to accept $y$ instead. To test for $x$ and $y$ being unranked, we need only see if the agent sometimes but not always accepts $x$ in exchange for $y$, and sometimes but not always accepts $y$ in exchange for $x$. Outcome-rational agents with substantively incomplete preferences must sometimes refuse to exchange unranked bundles, since otherwise they could be manipulated in the manner of Theorem 3; for simple choice environments, SQM furnishes the prime example of the optimality advantage of such refusals to trade. Formally, one checks $x \perp y$ by seeing if there exist both an $S^{i}$ such that $\{x, y\} \subset S^{i}, x \in \gamma^{i}$, and $y \notin \gamma^{i}$ and a $S^{k}$ such that $\{x, y\} \subset S^{k}, y \in \gamma^{k}$, and $x \notin \gamma^{k}$. Thus, by rejecting the traditional interpretation of revealed preferences-which imputes a preference judgment to all cases when a bundle $x$ is chosen over a bundle $y$-we can distinguish empirically among strict preference, weak preference, and the absence of preference. Rather it is the analytical strategy of defining a separate preference relation for each endowment vector that restricts the set of empirical tests.

The above empirical distinction between incompleteness and indifference presumes that since there is no drawback to exchanging one bundle for an indifferent bundle, rational agents will not hesitate to do so. If agents nevertheless do hesitate, there is a further empirical test. When two bundles are indifferent, a money or commodity bonus added to one of the bundles, no matter how small, should (given monotonicity) make that bundle strictly preferred. Agents with incomplete preferences, on the other hand, have rational grounds for refusing to swap unranked bundles, and there is no reason why adding a small amount of money or goods to one option will induce an agent to choose it.

Given the evidence, some aspect of the classical rationality model must give way. Behavioral models take the relatively drastic step of dissociating the choices made at different dates. Such a radical move is unnecessary. We can continue to assume that preferences are unchanging through time by dropping the assumption that unchosen bundles are necessarily dispreferred to chosen bundles. Psychological preference judgments can then stay fixed across time, which allows for welfare analysis and a larger set of empirical tests.

\subsection{Choice vs. preference as a primitive}

Although economic decision-makers can either be described through their psychological preferences or their choice behavior, our results provide grounds for using preferences rather than choice as primitive. Theorems 3 and 4 jointly demonstrate that summarizing choice behavior with a conventional choice function (or a conventional revealed preference relation) artificially constrains outcome-rational agents to choose transitively. Hence, 
if choice is taken as primitive, extended choice functions, which are relatively complicated objects, must be used to describe behavior. On the other hand, if psychological preference is the primitive of decision theory, a single binary relation can still adequately represent an agent: the set of outcome-rational extended choice functions can always be derived as necessary (if preferences are transitive). So a simpler, more concise theory emerges.

But if an extended choice function $\gamma$ is taken as primitive, and if $\gamma$ is assumed to obey some preference relation $\succcurlyeq$, then we can deduce $\succcurlyeq$ from $\gamma$. In this sense, the two starting points are interchangeable. Once $\succcurlyeq$ is inferred from $\gamma$, one can check whether $\gamma$ is outcome-rational. Thus, not only is incompleteness testable, so is outcome rationality.

\section{Acknowledgments}

I thank Drew Fudenberg, Steffen Huck, Peter Klibanoff, David Laibson, Klaus Nehring, and David Pearce for helpful comments and conversations. The paper has benefitted substantially from the detailed suggestions of two referees and an associate editor.

\section{Appendix A}

Proof of Theorem 4. Let $x, y$, and $z$ be such that $x \perp y, y \perp z$, and $z \succ x$. Let $\Sigma^{\prime} \subset \Sigma$ be the set of choice sequences defined by $\sigma^{\prime} \in \Sigma^{\prime}$ if and only if $S^{1 \prime}=\{x, y\}$ and $S^{2 \prime}=$ $\{x, y, z\}$. For any $\sigma^{\prime} \in \Sigma^{\prime}$, set $\gamma^{1}\left(\sigma^{\prime}\right)=\{x, y\}$ and $\gamma^{2}\left(\sigma^{\prime}\right)=\{y, z\}$. Hence $x R y$ and $y R z$. Since the $\gamma$ we construct is outcome-rational, for all $\sigma$ we have $x \notin \gamma^{t}(\sigma)$ whenever $z \in S^{t}$. Thus $z P x$ and hence $\gamma$ is verifiably intransitive.

It remains to define $\gamma$ over the remainder of its domain, and to show that $\gamma$ is measurable, obeys $\succcurlyeq$, and is outcome-rational. For $\sigma \notin \Sigma^{\prime}$, these tasks are mostly routine. The delicate parts are defining $\gamma$ on $\sigma^{\prime} \in \Sigma^{\prime}$ and showing that outcome rationality obtains for these $\sigma^{\prime}$.

To define $\gamma$, we utilize several binary relations. Let $X / \sim$ denote the indifference classes of $\succcurlyeq$, and, for any $a \in X$, let $I(a) \in X / \sim$ denote the indifference class that contains $a$. Define $\succ_{I}$ on $X / \sim$ by $I(a) \succ_{I} I(b)$ if and only if $a \succ b$. We first specify binary relations to define $\gamma$ for $\sigma$ such that $S^{1} \neq\{x, y\}$. For any $a, b \in X$ such that $a \perp b$, define $\succ_{a, b}$ on $X / \sim$ by $J \succ_{a, b} K$ if and only if $J \succ_{I} K$ or $J=I(a)$ and $K=I(b)$. It is easy to confirm that $\succ_{a, b}$ is acyclic. Hence $\succ_{a, b}$, the transitive closure of $\succ_{a, b}$, is irreflexive and thus satisfies $J \succ_{a, b} K \Rightarrow J \succ_{a, b} K$, i.e., $\succ_{a, b} \supset \succ_{a, b}$. Since $\succ_{a, b}$ is irreflexive and transitive, we may apply Szpilrajn's theorem, which states that any transitive irreflexive binary relation $\succ$ may be extended to a strict order $\succ^{*}$ (i.e., a transitive, asymmetric, and weakly connected binary relation), meaning that $\alpha \succ \beta$ implies $\alpha \succ^{*} \beta$. See Fishburn (1970) for a proof. So may extend $\bar{\succ}_{a, b}$ to a strict order, say $\succ_{a, b}^{*}$, on $X / \sim$. The relation $\succ_{a, b}^{*}$ contains $\bar{\succ}_{a, b}$ and hence $\succ_{a, b}$. We comment on our use of Szpilrajn's theorem following the proof.

For any binary relation $Z$ defined on a family of sets $\mathcal{P}$ that partition $X$, let $c_{Z}^{t}(\sigma)$ denote $\left\{u \in S^{t}\right.$ : not $p(\hat{u}) Z p(u)$ for all $\left.\hat{u} \in S^{t}\right\}$, where $p(u)$ denotes the cell of $\mathcal{P}$ to which $u$ belongs. If $\sigma$ is such that $S^{1}=\{a, b\} \neq\{x, y\}$ and $a \perp b$, we set $\gamma^{t}(\sigma)=c_{\succ_{a, b}^{*}}^{t}(\sigma)$ for all $t \geqslant 1$. If $\sigma$ is such that $S^{1} \supset\{a, b\} \neq\{x, y\}, a \perp b$, and \# $S^{1}=3$, let $\gamma^{t}(\sigma)=$ 
$c_{\succ_{b, a}^{*}}^{t}(\sigma)$ for all $t \geqslant 1$. For all other $\sigma$ such that $S^{1} \neq\{x, y\}$, we use an arbitrary strict order extension of $\succ_{I}$, say $\succ^{*}$, and set $\gamma^{t}(\sigma)=c_{\succ^{*}}^{t}(\sigma)$ for all $t \geqslant 1$. Note that for all $\sigma$ such that $S^{1} \neq\{x, y\}$, the mapping $\gamma^{t}(\sigma)$ depends only on $S^{1}$. So $\gamma(\sigma)$ trivially satisfies measurability for these $\sigma$. Also, if $a \perp b$ and $\{a, b\} \neq\{x, y\}$ then $\gamma$ is such that $a R b$ and $b R a$. We have singled out the $\# S^{1}=3$ case only as a convenient way to ensure $b R a$.

We turn to $\sigma$ such that $S^{1}=\{x, y\}$. Consider first cases where $S^{2} \neq\{x, y, z\}$. Let $I(x, y)$ denote $I(x) \cup I(y)$, and define $\succ_{I(x, y)}$ a binary relation on $\mathbf{Y}=X / \sim \cup\{I(x, y)\} \backslash$ $\{I(x), I(y)\}$, by $J \succ_{I(x, y)} K$ if and only if one of the following three statements holds:

(i) $J \succ_{I} K$ and $J, K \notin\{I(x), I(y)\}$,

(ii) $J=I(x, y)$ and $I(x) \succ_{I} K$ or $I(y) \succ_{I} K$, or

(iii) $K=I(x, y)$ and $J \succ_{I} I(x)$ or $J \succ_{I} I(y)$.

To see that $\succ_{I(x, y)}$ is acyclic, suppose the contrary and observe that one of two statements must then hold:

(1) $I(x, y) \succ_{I(x, y)} \cdots \succ_{I(x, y)} I(x, y)$, or

(2) $I(a) \succ_{I(x, y)} \cdots \succ_{I(x, y)} I(x, y) \succ_{I(x, y)} \cdots \succ_{I(x, y)} I(a)$,

where $I(x, y)$ is not an indifference class in the ellipses. If we replace each $\succ_{I(x, y)}$ in (1) by $\succ_{I}$, we may either replace both $I(x, y)$ 's in (1) by $I(x)$ or $I(y)$, which would violate the acyclicity of $\succ_{I}$, or we may replace one $I(x, y)$ by $I(x)$ and the other by $I(y)$, in which case the transitivity of $\succ_{I}$ implies a violation of $x \perp y$. As for (2), we can infer $I(a) \succ_{I} \cdots \succ_{I} I(u)$ and $I(w) \succ_{I} \cdots \succ_{I} I(a)$, where $u, w \in\{x, y\}$. If $u=w$, we have violation of the acyclicity of $\succ_{I}$, and if $u \neq w$, a violation of $x \perp y$. So $\succ_{I(x, y)}$ is acyclic and $\bar{\succ}_{I(x, y)}$, the transitive closure of $\succ_{I(x, y)}$, is irreflexive. Applying Szpilrajn to $\bar{\succ}_{I(x, y)}$, we obtain a strict order $\succ_{I(x, y)}^{*}$ over $\mathbf{Y}$ that contains $\succ_{I(x, y)}$. For $\sigma$ such that $S^{1}=\{x, y\}$ and $S^{2} \neq\{x, y, z\}$, set $\gamma^{t}(\sigma)=c_{\succ_{I(x, y)}^{t}}^{t}(\sigma)$ for $t \geqslant 1$.

To finish the specification of $\gamma$, consider the $\Sigma^{\prime}$ sequences. First, define $\succ_{I(y, z)}$ as in the last paragraph except that $y$ and $z$ take the role of $x$ and $y$. Formally, $I(y, z)$ denotes $I(y) \cup I(z)$ and $\succ_{I(y, z)}$, a binary relation on $\mathbf{Z}=X / \sim \cup\{I(y, z)\} \backslash\{I(y), I(z)\}$, is defined by $J \succ_{I(y, z)} K$ if and only if $\left(J \succ_{I} K\right.$ and $\left.J, K \notin\{I(y), I(z)\}\right)$ or $(J=I(y, z)$ and $I(y) \succ_{I} K$ or $\left.I(z) \succ_{I} K\right)$ or $\left(K=I(y, z)\right.$ and $J \succ_{I} I(y)$ or $\left.J \succ_{I} I(z)\right)$. As in the case of $\succ_{I(x, y)}, \succ_{I(y, z)}$ is acyclic.

Next, define $\succ_{\sigma^{\prime}}$, a binary relation on $\mathbf{Z}$, by $J \succ_{\sigma^{\prime}} K$ if and only if $J \succ_{I(y, z)} K$ or $J=I(x)$ and $K \in \mathbf{L} \equiv\{L \in X / \sim: L=I(w)$ for some $w \in X$ such that $y \succ w\}$. As indicated in the text, this is the key step that ensures outcome rationality, as we will see at the end of the proof. Let $\bar{\succ}_{\sigma^{\prime}}$ denote the transitive closure of $\succ_{\sigma^{\prime}}$. To conclude that $J \succ_{\sigma^{\prime}} K$ implies $J \bar{\succ}_{\sigma^{\prime}} K$ and that $\succ_{\sigma^{\prime}}$ is irreflexive, we show that $\succ_{\sigma^{\prime}}$ is acyclic. Given the acyclicity of $\succ_{I(y, z)}$, if $\succ_{\sigma^{\prime}}$ has a cycle then one of the following two conditions must hold:

(i) $I(x) \succ_{\sigma^{\prime}} \cdots \succ_{\sigma^{\prime}} I(x)$, or

(ii) $J \succ_{\sigma^{\prime}} \cdots \succ_{\sigma^{\prime}} I(x) \succ_{\sigma^{\prime}} \cdots \succ_{\sigma^{\prime}} J$ for some $J \in \mathbf{Z}$, 
where $I(x)$ is not one of the indifference classes in the ellipses. Except for the $\succ_{\sigma^{\prime}}$ that lies between $I(x)$ and its neighbor to the right, which we label $W$, each $\succ_{\sigma^{\prime}}$ in (i) or (ii) may be replaced by $\succ_{I(y, z)}$. Since the acyclicity of $\succ_{I(y, z)}$ implies that we may not replace every $\succ_{\sigma^{\prime}}$ by $\succ_{I(y, z)}, W$ must be an element of $\mathbf{L}$ and so $I(y) \succ_{I} W$. In case (i), we therefore have $W \succ_{I(y, z)} \cdots \succ_{I(y, z)} I(x)$. To see that $I(y, z)$ cannot be an indifference class in the preceding ellipsis, suppose the contrary and consider the leftmost occurrence of $I(y, z)$. Since $W \neq I(y, z)$, we then have either $W \succ_{I} \cdots \succ_{I} I(y)$ or $W \succ_{I} \cdots \succ_{I} I(z)$. Combined with $I(y) \succ_{I} W$, the acyclicity of $\succ_{I}$ yields a contradiction for the first possibility, while the transitivity of $\succ_{I}$ and the fact that $y \perp z$ yields a contradiction for the second. We conclude that $W \succ_{I} \cdots \succ_{I} I(x)$, and therefore $W \succ_{I} I(x)$. Since $I(y) \succ_{I} W$, the transitivity of $\succ_{I}$ implies $I(y) \succ_{I} I(x)$, which contradicts $x \perp y$. In case (ii), we have

$$
J \succ_{I(y, z)} \cdots \succ_{I(y, z)} I(x) \succ_{\sigma^{\prime}} W \succ_{I(y, z)} \cdots \succ_{I(y, z)} J .
$$

Using the same reasoning as in case (i), $I(y, z)$ cannot be an indifference class in the second ellipsis. So $W \succ_{I} J$. Since $I(y) \succ_{I} W$, we have $I(y) \succ_{I} J$ and therefore $J \neq$ $I(y, z)$. Hence $I(y, z)$ cannot be an indifference class in the first ellipsis. So $J \succ_{I} I(x)$ and therefore $W \succ_{I} I(x)$. As in case (i), $I(y) \succ_{I} W$ and the transitivity of $\succ_{I}$ again contradict $x \perp y$.

We conclude that $\succ_{\sigma^{\prime}}$ is acyclic and that $\bar{\succ}_{\sigma^{\prime}}$ is irreflexive. So, by Szpilrajn, there exists a strict order $\succ_{\sigma^{\prime}}^{*}$ over $\mathbf{Z}$ that contains $\succ_{\sigma^{\prime}}$. For $\sigma^{\prime} \in \Sigma^{\prime}$, set $\gamma^{t}(\sigma)=c_{\succ_{\sigma^{\prime}}^{*}}^{t}(\sigma)$ for $t \geqslant 3$. Since we specified $\gamma^{1}\left(\sigma^{\prime}\right)$ and $\gamma^{2}\left(\sigma^{\prime}\right)$ in the first paragraph, $\gamma$ is now well defined.

Recall that the mapping $\gamma^{t}(\sigma), t \geqslant 1$, for $\sigma$ such that $S^{1} \neq\{x, y\}$ depends only on $S^{1}$. For all $\sigma$ such that $S^{1}=\{x, y\}, \gamma^{1}(\sigma)=\{x, y\}$. If $\sigma^{\prime} \in \Sigma^{\prime}$, then the mapping $\gamma^{t}\left(\sigma^{\prime}\right)$, evaluated at $t \geqslant 2$, is determined according to $\succ_{\sigma^{\prime}}^{*}$ and hence does not depend on any $S^{t}$ with $t \geqslant 3$. If $S^{1}=\{x, y\}$ and $S^{2} \neq\{x, y, z\}, \gamma^{t}(\sigma)$, for $t \geqslant 2$, is determined according to $\succ_{I(x, y)}^{*}$ and hence also does not depend on any $S^{t}$ with $t \geqslant 3$. So $\gamma$ is measurable.

We confirm that $\gamma$ satisfies (1) of Definition 4: $u \sim w, w \in \gamma^{t}(\sigma), u \in S^{t} \Rightarrow u \in \gamma^{t}(\sigma)$. Note that neither $\{x, y\}$ nor $\{x, y, z\}$ contain a pair of indifferent elements. So if $u \sim w$ and $u, w \in S^{t}$, then $\gamma^{t}(\sigma)=c_{Z}^{t}(\sigma)$ for some $Z \in\left\{\succ_{a, b}^{*}: a \perp b\right\} \cup\left\{\succ^{*}, \succ_{I(x, y)}^{*}, \succ_{\sigma^{\prime}}^{*}\right\}$. Since each $Z$ is an ordering over indifference classes (or unions of indifference classes), (1) is satisfied. As for (2), $u \perp w \Rightarrow u R w$, we earlier remarked that if $a \perp b$ and $\{a, b\} \neq\{x, y\}$ then $a R b$. Given that $\gamma^{1}\left(\sigma^{\prime}\right)=\{x, y\}$ for $\sigma^{\prime} \in \Sigma^{\prime}, x R y$ and $y R x$.

To show that $\gamma$ is outcome-rational, we use the following lemma.

Lemma. Given a complete and transitive binary relation $\tilde{R} \subset X \times X$, let $\tilde{\gamma}$ be defined by $\tilde{\gamma}^{t}(\sigma)=\left\{a \in S^{t}: a \tilde{R} b\right.$ for all $\left.b \in S^{t}\right\}$. If $t$ is linked to $\tau<t$ at $\sigma$ for $\tilde{\gamma}, s \in \tilde{\gamma}(\sigma)$, and $r \in S^{\tau}$, then $s \tilde{R} r$.

To prove the lemma, note that if $t$ is linked to $\tau$, there exists $a \in \gamma^{\tau}(\sigma)$ such that $a \in S^{t}$. By the definition of $\tilde{\gamma}, s \in \gamma^{t}(\sigma)$ implies $s \tilde{R} a$ and $a \in \gamma^{\tau}(\sigma)$ implies $a \tilde{R} r$ for $r \in S^{\tau}$. By the transitivity of $\tilde{R}, s \tilde{R} r$.

Consider first cases where $\sigma \notin \Sigma^{\prime}$. Let $R(\sigma)$ be defined by $u R(\sigma) w$ if and only if $I(u)=I(w)$ or $I(u) Z I(w)$ where $Z$ is $\succ_{a, b}^{*}$ if $S^{1}=\{a, b\} \neq\{x, y\}$ and $a \perp b ; \succ_{b, a}^{*}$ if $S^{1} \supset\{a, b\} \neq\{x, y\}, S^{1}=3$, and $a \perp b ; \succ_{I(x, y)}^{*}$ if $S^{1}=\{x, y\}$; and $\succ^{*}$ otherwise. Note 
that $R(\sigma)$ is transitive and $u \succ w$ implies $u P(\sigma) w$ (i.e., $u R(\sigma) w$ and not $w R(\sigma) u)$. Also, for all $t \geqslant 1, \gamma^{t}(\sigma)=\left\{u \in S^{t}: u R(\sigma) \hat{u}\right.$ for all $\left.\hat{u} \in S^{t}\right\}$. Let $\left\{t_{1}, t_{2}, \ldots, t_{m}\right\}$ be such that $t_{j}$ is linked to $t_{j-1}$ at $\sigma$ for $j \in\{2, \ldots, m\}$. Fix arbitrary $x^{1} \in S^{t_{1}}$ and, for $j \in\{2, \ldots, m\}$, $x^{j} \in \gamma^{t_{j}}(\sigma)$. The lemma implies, for $j \in\{2, \ldots, m\}$, that $x^{j} R(\sigma) x^{j-1}$, and so, by the transitivity of $R(\sigma), x^{m} R(\sigma) x^{1}$. Since $u \succ w$ implies not $w R(\sigma) u$, we conclude that not $x^{1} \succ x^{m}$. So $\gamma(\sigma)$ is outcome-rational when $\sigma \notin \Sigma^{\prime}$.

Now let $\sigma^{\prime} \in \Sigma^{\prime}$. Define $R\left(\sigma^{\prime}\right)$ by $u R\left(\sigma^{\prime}\right) w$ if and only if $I(u)=I(w)$ or $I(u) \succ_{\sigma^{\prime}}^{*}$ $I(w)$. As before, $R\left(\sigma^{\prime}\right)$ is transitive and $u \succ w$ implies $u P\left(\sigma^{\prime}\right) w$. Again let $\left\{t_{1}, t_{2}, \ldots, t_{m}\right\}$ be such that $t_{j}$ is linked to $t_{j-1}$ at $\sigma^{\prime}$ for $j \in\{2, \ldots, m\}$, fix arbitrary $x^{1} \in S^{t_{1}{ }^{\prime}}$ and, for $j \in\{2, \ldots, m\}, x^{j} \in \gamma^{t_{j}}\left(\sigma^{\prime}\right)$. Note that $\gamma^{t}\left(\sigma^{\prime}\right)=\left\{u \in S^{t^{\prime}}: u R\left(\sigma^{\prime}\right) \hat{u}\right.$ for all $\left.\hat{u} \in S^{t^{\prime}}\right\}$ for all $\sigma^{\prime}$ and all $t \geqslant 2$. Hence, if $t_{1} \geqslant 2$ then, by the lemma, $x^{j} R\left(\sigma^{\prime}\right) x^{j-1}$ for $j \in\{2, \ldots, m\}$. Hence $x^{m} R\left(\sigma^{\prime}\right) x^{1}$ and so not $x^{1} \succ x^{m}$. So suppose henceforth that $t_{1}=1$. Since $x$ is the element of $S^{1^{\prime}}=\{x, y\}$ that is minimal according to $R\left(\sigma^{\prime}\right)$, if $x^{1}=x$ then $x^{2} R\left(\sigma^{\prime}\right) x^{1}$. Hence, $x^{m} R\left(\sigma^{\prime}\right) x^{1}$ and not $x^{1} \succ x^{m}$. So assume henceforth that $x^{1}=y$. Since $t_{2}$ is linked to $t_{1}$, if $x \notin S^{t_{2}}$ then $y \in S^{t_{2}}$. So $x^{2} R\left(\sigma^{\prime}\right) x^{1}$ and therefore $x^{m} R\left(\sigma^{\prime}\right) x^{1}$ and not $x^{1} \succ x^{m}$. So assume that $x \in S^{t_{2}}$. If this last possibility constituted a failure of outcome rationality, then $y \succ x^{m}$. But the construction of $\succ_{\sigma^{\prime}}^{*}$ would then imply $I(x) \succ_{\sigma^{\prime}}^{*} I\left(x^{m}\right)$ and so $x P\left(\sigma^{\prime}\right) x^{m}$. Since $x \in S^{t_{2}}, x^{2} R\left(\sigma^{\prime}\right) x$ and hence $x^{m} R\left(\sigma^{\prime}\right) x$, a contradiction.

Note. Szpilrajn's theorem is a needlessly powerful-and nonconstructive-tool for the main point we wish to establish. For each $\sigma$ and $t$, the agent could determine $\gamma^{t}(\sigma)$ by assembling a binary relation $Z_{t}$ over the finite set $\bigcup_{t=1}^{t} S^{t}$ that possesses the properties of the relations $\succ_{a, b}^{*}, \succ^{*}, \succ_{I(x, y)}^{*}$ and $\succ_{\sigma^{\prime}}^{*}$ used in the proof and that extends $Z^{t-1}$. At each $t$, therefore, the agent's selection of $\gamma^{t}$ would be entirely constructive.

\section{References}

Anand, P., 1993. Foundations of Rational Choice Under Risk. Clarendon Press, Oxford.

Arrow, K., 1959. Rational choice functions and orderings. Economica 26, 121-127.

Aumann, R., 1962. Utility theory without the completeness axiom. Econometrica 30, 445-462.

Bewley, T., 1986. Knightian decision theory: Part 1. Discussion paper No. 807. Cowles Foundation, New Haven. Bewley, T., 1987. Knightian decision theory: Part 2. Discussion paper No. 835. Cowles Foundation, New Haven. Camerer, C., 1995. Individual decision making. In: Kagel, J., Roth, A. (Eds.), Handbook of Experimental Economics.

Cubitt, R., Sugden, R., 2001. On money pumps. Games Econ. Behav. 37, 121-160.

Danan, E., 2001. Behavioral foundations of incomplete preferences. Mimeo. Université de Paris 1, Paris.

Davidson, D., McKinsey, J., Suppes, P., 1955. Outlines of a formal theory of value, I. Philos. Sci. 22, 140-160.

Dubra, J., Maccheroni, F., Ok, E., 2001. Expected utility theory without the completeness axiom. J. Econ. Theory $115,118-133$.

Eliaz, K., Ok, E., 2002. Indifference or indecisiveness: Choice theoretic foundations of incomplete preferences. Mimeo. New York University, New York.

Fishburn, P., 1970. Utility Theory for Decision Making. Wiley, New York.

Fishburn, P., 1988. Nonlinear Preference and Utility Theory. Johns Hopkins, Baltimore.

Kahneman, D., Knetsch, J., Thaler, R., 1990. Experimental tests of the endowment effect and the Coase theorem. J. Polit. Economy 98, 1325-1348.

Knetsch, J., 1989. The endowment effect and evidence of nonreversible indifference curves. Amer. Econ. Rev. 79, 1277-1284. 
Levi, I., 1986. Hard Choices: Decision Making Under Unresolved Conflict. Cambridge Univ. Press, Cambridge. Mandler, M., 1998. The economics of incomplete preferences. Mimeo. Harvard University, Cambridge.

Mandler, M., 2004. Status quo maintenance reconsidered: changing or incomplete preferences? Econ. J. 114, 518-535.

Mas-Colell, A., Whinston, M., Green, J., 1995. Microeconomic Theory. Oxford Univ. Press, New York.

Nehring, K., 1997. Rational choice and revealed preference without binariness. Soc. Choice Welfare 14, 403-425.

Ok, E., 2002. Utility representation of an incomplete preference relation. J. Econ. Theory 104, 429-449.

Peleg, B., 1970. Utility functions for partially ordered topological spaces. Econometrica 38, 93-96.

Rabin, M., 1998. Psychology and economics. J. Econ. Lit. 36, 11-46.

Raiffa, H., 1968. Decision Analysis. Addison-Wesley, Reading, MA.

Richter, M., 1966. Revealed preference theory. Econometrica 34, 635-645.

Samuelson, W., Zeckhauser, R., 1988. Status quo bias in decision making. J. Risk Uncertainty 1, 7-59.

Sen, A., 1973. Behaviour and the concept of preference. Economica 40, 241-259.

Sen, A., 1982. Introduction. In: Sen, A. (Ed.), Choice, Welfare and Measurement. Blackwell, Oxford, pp. 1-38. Sen, A., 1997. Maximization and the act of choice. Econometrica 65, 745-780.

Thaler, R., 1980. Toward a positive theory of consumer choice. J. Econ. Behav. Organ. 1, 39-60.

Tversky, A., Kahneman, D., 1991. Loss aversion in riskless choice: a reference-dependent model. Quart. J. Econ. 106, 1039-1061. 